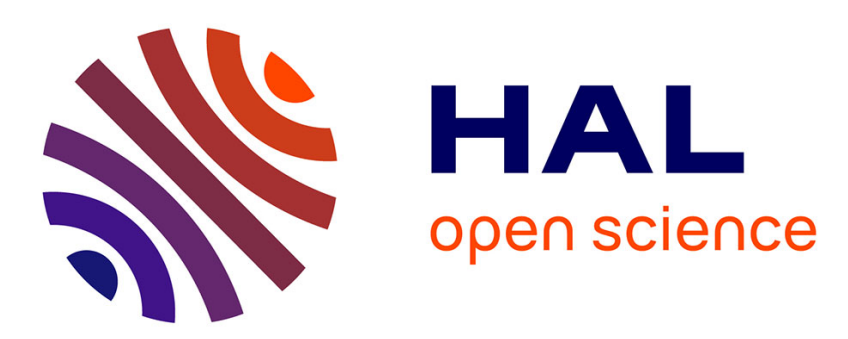

\title{
Symmetrization of functions and principal eigenvalues of elliptic operators
}

François Hamel, Nikolai Nadirashvili, Emmanuel Russ

\section{To cite this version:}

François Hamel, Nikolai Nadirashvili, Emmanuel Russ. Symmetrization of functions and principal eigenvalues of elliptic operators. Séminaire Laurent Schwartz - EDP et applications, 2012, pp.15. 10.5802/slsedp.19 . hal-01286474

\section{HAL Id: hal-01286474 \\ https://hal.science/hal-01286474}

Submitted on 10 Mar 2016

HAL is a multi-disciplinary open access archive for the deposit and dissemination of scientific research documents, whether they are published or not. The documents may come from teaching and research institutions in France or abroad, or from public or private research centers.
L'archive ouverte pluridisciplinaire HAL, est destinée au dépôt et à la diffusion de documents scientifiques de niveau recherche, publiés ou non, émanant des établissements d'enseignement et de recherche français ou étrangers, des laboratoires publics ou privés. 


\title{
Symmetrization of functions and principal eigenvalues of elliptic operators
}

\author{
François Hamel ${ }^{\mathrm{a}}$, Nikolai Nadirashvili ${ }^{\mathrm{b}}$ and Emmanuel Russ ${ }^{\mathrm{c} *}$ \\ a Aix-Marseille Université \& Institut Universitaire de France \\ LATP, Avenue Escadrille Normandie-Niemen, 13397 Marseille Cedex 20, France \\ b CNRS, LATP, 39 rue F. Joliot-Curie, 13453 Marseille Cedex 13, France \\ ${ }^{\mathrm{c}}$ Université Joseph Fourier, Institut Fourier, 100 rue des Maths, BP 74, 38402 St-Martin d'Hères \\ X-EDP Seminar 2011
}

\begin{abstract}
In this paper, we consider shape optimization problems for the principal eigenvalues of second order uniformly elliptic operators in bounded domains of $\mathbb{R}^{n}$. We first recall the classical Rayleigh-Faber-Krahn problem, that is the minimization of the principal eigenvalue of the Dirichlet Laplacian in a domain with fixed Lebesgue measure. We then consider the case of the Laplacian with a bounded drift, that is the operator $-\Delta+v \cdot \nabla$, for which the minimization problem is still well posed. Next, we deal with more general elliptic operators $-\operatorname{div}(A \nabla)+v \cdot \nabla+V$, for which the coefficients fulfill various pointwise, integral or geometric constraints. In all cases, some operators with radially symmetric coefficients in an equimeasurable ball are shown to have smaller principal eigenvalues. Whereas the Faber-Krahn proof relies on the classical Schwarz symmetrization, another type of symmetrization is defined to handle the case of general (possibly non-symmetric) operators.
\end{abstract}

\section{The Rayleigh-Faber-Frahn theorem}

Let $n \geq 1$ be any integer. For any bounded domain (non-empty connected set) $\Omega \subset \mathbb{R}^{n}$, let

$$
0<\lambda_{1}(\Omega)<\lambda_{2}(\Omega) \leq \ldots \leq \lambda_{k}(\Omega) \leq \ldots
$$

be the sequence of ordered eigenvalues of the Laplacian operator $-\Delta$ under Dirichlet boundary condition. Throughout the paper, we only consider smooth domains, that is domains

*The authors are supported by the French "Agence Nationale de la Recherche" in the project PREFERED (ANR 08-BLAN-0313). 
whose boundary is of class $C^{2}$. The principal eigenvalue $\lambda_{1}(\Omega)$, which is positive and is the smallest one (notice that all eigenvalues are real since the Laplacian is symmetric), is simple and characterized by the existence of a signed eigenfunction. That is, there is a function $\varphi$ belonging to the Sobolev spaces $W^{2, p}(\Omega)$ for all $1 \leq p<+\infty$, such that

$$
\left\{\begin{aligned}
-\Delta \varphi & =\lambda_{1}(\Omega) \varphi & & \text { a.e. in } \Omega, \\
\varphi & >0 & & \text { in } \Omega, \\
\varphi & =0 & & \text { on } \partial \Omega .
\end{aligned}\right.
$$

Question 1.1 Let $m>0$. Among all the $C^{2}$ bounded domains $\Omega \subset \mathbb{R}^{n}$ with Lebesgue measure $m$, for which $\Omega$ does $\lambda_{1}(\Omega)$ reach its infimum?

Notice immediately that, if the infimum is replaced by the supremum, then the question is ill posed in any dimension $n \geq 2$ since it is easy to find a sequence of thiner and thiner domains $\left(\Omega_{k}\right)_{k \in \mathbb{N}}$ with Lebesgue measure $m$ and for which $\lambda_{1}\left(\Omega_{k}\right) \rightarrow+\infty$ as $k \rightarrow+\infty$.

For any bounded domain $\Omega$, let $\Omega^{*}$ be the Euclidean ball with enter 0 and having the same Lebesgue measure as $\Omega$, that is $\left|\Omega^{*}\right|=|\Omega|$, where $|A|$ denotes the Lebesgue measure of any measurable subset $A \subset \mathbb{R}^{n}$. The answer to Question 1.1 is given in the following theorem.

Theorem $1.2[13,19,20,23]$ The infimum of $\lambda_{1}(\Omega)$ among all bounded $C^{2}$ domains of $\mathbb{R}^{n}$ is reached when $\Omega$ is a Euclidean ball, and only in this case. In other words,

$$
\lambda_{1}(\Omega) \geq \lambda_{1}\left(\Omega^{*}\right)
$$

and the equality holds if and only if $\Omega=\Omega^{*}$ up to a shift.

Theorem 1.2 was conjectured by Rayleigh [24] in 1894 when $n=2$, that is the disk is the (unique) domain which minimizes $\lambda_{1}(\Omega)$ over all the domains $\Omega$ with given area. The proof of the inequality (1.2) was given independently by Faber [13] in 1923 and Krahn [19] in 1925 when $n=2$, and later by Krahn [20] in 1926 for all $n$. It goes through a variational formulation for $\lambda_{1}(\Omega)$ :

$$
\lambda_{1}(\Omega)=\min _{\varphi \in H_{0}^{1}(\Omega) \backslash\{0\}} \frac{\int_{\Omega}|\nabla \varphi(x)|^{2} d x}{\int_{\Omega}|\varphi(x)|^{2} d x},
$$

which holds since $-\Delta$ is symmetric on $L^{2}(\Omega)$. Notice that the minimum is reached by the principal eigenfunction $\varphi$ solving (1.1). The proof also makes use of the Schwarz symmetrization. Namely, if $u: \Omega \rightarrow \mathbb{R}$ is measurable, one can define the Schwarz symmetrization $u^{*}$ of $u$, as the function $u^{*}: \Omega^{*} \rightarrow \mathbb{R}$ which is radially symmetric, non-increasing with respect to the Euclidean norm $|x|$ for $x \in \Omega^{*}$ and is such that

$$
|\{x \in \Omega ; u(x)>t\}|=\left|\left\{x \in \Omega^{*} ; u^{*}(x)>t\right\}\right|
$$


for all $t \in \mathbb{R}$. If $u \in H_{0}^{1}(\Omega)$, then $|u|^{*} \in H_{0}^{1}\left(\Omega^{*}\right)$. Furthermore, $\left\||u|^{*}\right\|_{L^{2}\left(\Omega^{*}\right)}=\|u\|_{L^{2}(\Omega)}$ (since both $|u|$ and $|u|^{*}$ have by definition the same distribution function) and $\left\|\nabla|u|^{*}\right\|_{L^{2}\left(\Omega^{*}\right)} \leq$ $\|\nabla u\|_{L^{2}(\Omega)}$, see [23] (the proof of this inequality comes from the classical isoperimetric inequality, from the co-area formula and the Cauchy-Schwarz and Young inequalities, as formulated in $[13,19,20])$. One therefore obtains

$$
\lambda_{1}(\Omega)=\frac{\int_{\Omega}|\nabla \varphi(x)|^{2} d x}{\int_{\Omega}|\varphi(x)|^{2} d x} \geq \frac{\int_{\Omega^{*}}\left|\nabla \varphi^{*}(x)\right|^{2} d x}{\int_{\Omega^{*}}\left|\varphi^{*}(x)\right|^{2} d x} \geq \lambda_{1}\left(\Omega^{*}\right) .
$$

The proof of the sharpness of (1.2), that is the study of the case of equality, is more intricate and requires some smoothness assumption on $\Omega$, see [23]. The now-called Faber-Krahn inequality (1.2) yields an "explicit" lower bound for $\lambda_{1}(\Omega)$ :

$$
\lambda_{1}(\Omega) \geq|\Omega|^{-2 / n} \alpha_{n}^{2 / n}\left(j_{n / 2-1,1}\right)^{2},
$$

where $\alpha_{n}=\pi^{n / 2} / \Gamma(n / 2+1)$ is the Lebesgue measure of the Euclidean unit ball in $\mathbb{R}^{n}$ and $j_{m, 1}$ denotes the first positive zero of the Bessel function $J_{m}$ (notice that the right-hand side of the above inequality only depends on $n$ and $|\Omega|$ ).

For some surveys on optimization problems of the eigenvalues of the Laplacian and on further isoperimetric inequalities, we refer to $[6,17,25]$ (see also $[9,16]$ for some results on the other eigenvalues of the Laplacian, $[4,10,22]$ for some results on some functions of the eigenvalues, $[5,21]$ for the minimization of the first eigenvalue of $\Delta^{2}$ in dimensions 2 and 3 , and $[8,11,12,28]$ for some results on the eigenvalues of the Laplacian under other boundary conditions). All the proofs rely on the symmetry of the operator and on some Rayleigh variational formulation of the eigenvalues. It is thus natural to seek for generalizations of (1.2) when $-\Delta$ is replaced by a more general second order elliptic operator, possibly nonsymmetric. We first focus in Section 2 on a rather simple (but non-symmetric) situation, that is the case of the Laplacian with drift. We next deal in Section 3 with the case of more general second order elliptic operators.

\section{The case of the Laplacian with drift}

Let first $\Omega$ be a fixed $C^{2}$ bounded domain of $\mathbb{R}^{n}$ with $n \geq 1$. If $1 \leq p \leq+\infty$ and $v: \Omega \rightarrow \mathbb{R}^{n}$ is measurable, we say that $v \in L^{p}\left(\Omega, \mathbb{R}^{n}\right)$ if $|v| \in L^{p}(\Omega)(|v|$ denotes the Euclidean norm of $v)$, and we write $\|v\|_{p}=\|v\|_{L^{p}\left(\Omega, \mathbb{R}^{n}\right)}:=\|v\|_{L^{p}(\Omega)}$. If $v \in L^{\infty}\left(\Omega, \mathbb{R}^{n}\right)$, then the operator $-\Delta+v \cdot \nabla$ under Dirichlet boundary condition has a principal eigenvalue $\lambda_{1}(\Omega, v)$ associated with a corresponding eigenfunction $\varphi$ belonging to the Sobolev spaces $W^{2, p}(\Omega)$ for all $1 \leq p<+\infty$, such that

$$
\left\{\begin{aligned}
-\Delta \varphi+v \cdot \nabla \varphi & =\lambda_{1}(\Omega, v) \varphi & & \text { a.e. in } \Omega, \\
\varphi & >0 & & \text { in } \Omega, \\
\varphi & =0 & & \text { on } \partial \Omega,
\end{aligned}\right.
$$


see [7]. Furthermore, $\lambda_{1}(\Omega, v)$ is simple, real, positive (from the maximum principle) and all other eigenvalues have a real part which is larger than $\lambda_{1}(\Omega, v)$.

Question 2.1 Let $\Omega$ be a fixed $C^{2}$ bounded domain of $\mathbb{R}^{n}$ and let $\tau \geq 0$ be a fixed nonnegative real number. What can be said about

$$
\lambda^{-}(\Omega, \tau):=\inf _{v \in L^{\infty}\left(\Omega, \mathbb{R}^{n}\right),\|v\|_{\infty} \leq \tau} \lambda_{1}(\Omega, v)
$$

and

$$
\lambda^{+}(\Omega, \tau):=\sup _{v \in L^{\infty}\left(\Omega, \mathbb{R}^{n}\right),\|v\|_{\infty} \leq \tau} \lambda_{1}(\Omega, v) ?
$$

It follows from some general bounds on the principal eigenvalues [7] that these two quantities are real numbers. Furthermore, it turns out that the optimizing vector fields exist and are unique, as the following theorem from $[14,15]$ shows.

Theorem $2.2[14,15]$ Let $\Omega$ be a $C^{2}$ bounded domain of $\mathbb{R}^{n}$ and let $\tau \geq 0$. Then there exist two unique vector fields $v^{ \pm} \in L^{\infty}\left(\Omega, \mathbb{R}^{n}\right)$ such that $\left\|v^{ \pm}\right\|_{\infty} \leq \tau$ and

$$
\lambda_{1}\left(\Omega, v^{ \pm}\right)=\lambda^{ \pm}(\Omega, \tau) .
$$

Furthermore, $\left|v^{ \pm}(x)\right|=\tau$ for a.e. $x \in \Omega$ and the principal eigenfunctions $\varphi^{ \pm}$of the operators $-\Delta+v^{ \pm} \cdot \nabla$ satisfy $v^{ \pm} \cdot \nabla \varphi^{ \pm}= \pm \tau\left|\nabla \varphi^{ \pm}\right|$a.e. in $\Omega$, that is the functions $\varphi^{ \pm}$then solve the following nonlinear problems

$$
-\Delta \varphi^{ \pm} \pm \tau\left|\nabla \varphi^{ \pm}\right|=\lambda^{ \pm}(\Omega, \tau) \varphi^{ \pm} \text {a.e. in } \Omega \text {. }
$$

When $\Omega$ is a ball (without loss of generality, one can then assume that it is centered at the origin 0 ), one can give the explicit expression of the optimizing fields $v^{ \pm}$. For that, we need an auxiliary notation: for all $x \in \mathbb{R}^{n} \backslash\{0\}$, set

$$
e_{r}(x)=\frac{x}{|x|}
$$

Theorem $2.3[14,15]$ Assume that $\Omega$ is an open Euclidean ball of center 0 and let $\tau \geq 0$ be fixed. Then $v^{ \pm}=\mp \tau e_{r}$, and the functions $\varphi^{ \pm}$are radially decreasing in $\Omega$.

Remark 2.4 The above optimization results do not hold anymore without the bound on the $L^{\infty}$ norm of the vector field $v$. More precisely, $\sup _{v \in L^{\infty}\left(\Omega, \mathbb{R}^{n}\right)} \lambda_{1}(\Omega, v)=+\infty$ and $\inf _{v \in L^{\infty}\left(\Omega, \mathbb{R}^{n}\right)} \lambda_{1}(\Omega, v)=0$ is not reached. Furthermore, the results do not hold anymore in general when the $L^{\infty}$ constraints are replaced by other kinds of constraints. For instance, it is immediate to see that, for any $1<p<n$ and $\tau \in[0,+\infty)$, the infimum $\inf _{v \in L^{p}\left(\Omega, \mathbb{R}^{n}\right),\|v\|_{p} \leq \tau} \lambda_{1}(\Omega, v)$ is equal to 0 and is then not reached.

In view of Theorem 2.2 and of (1.2), it is natural to let now both $\Omega$ and $v$ vary. 
Question 2.5 Let $n \geq 1, m>0$ and $\tau \geq 0$ be fixed. Among all the $C^{2}$ bounded domains $\Omega \subset \mathbb{R}^{n}$ with $|\Omega|=m$ and all the vector fields $v \in L^{\infty}\left(\Omega, \mathbb{R}^{n}\right)$ such that $\|v\|_{\infty}=\|v\|_{L^{\infty}\left(\Omega, \mathbb{R}^{n}\right)} \leq \tau$, for which pair $(s)(\Omega, v)$ does $\lambda_{1}(\Omega, v)$ reach its infimum ?

Notice immediately that the supremum is $+\infty$ (take $v=0$ and remember that $\left.\sup _{|\Omega|=m} \lambda_{1}(\Omega)=+\infty\right)$. The answer to Question 2.5 is given in the following theorem.

Theorem 2.6 $[14,15]$ Let $\Omega \subset \mathbb{R}^{n}$ be a $C^{2}$ bounded domain, let $\tau \geq 0$ and let $v \in$ $L^{\infty}\left(\Omega, \mathbb{R}^{n}\right)$ with $\|v\|_{L^{\infty}\left(\Omega, \mathbb{R}^{n}\right)} \leq \tau$. Then

$$
\lambda_{1}(\Omega, v) \geq \lambda_{1}\left(\Omega^{*}, \tau e_{r}\right)
$$

and the equality holds if and only if, up to translation, $\Omega=\Omega^{*}$ and $v=\tau e_{r}$.

The previous result can be reformulated as a Faber-Krahn type inequality. Namely, for all $n \geq 1$, there is a function $F_{n}:(0,+\infty) \times[0,+\infty) \rightarrow(0,+\infty)$ such that

$$
\lambda_{1}(\Omega, v) \geq F_{n}\left(|\Omega|,\|v\|_{\infty}\right)=\lambda_{1}\left(\Omega^{*},\|v\|_{\infty} e_{r}\right)
$$

for every $C^{2}$ bounded domain $\Omega \subset \mathbb{R}^{n}$ and every vector field $v \in L^{\infty}\left(\Omega, \mathbb{R}^{n}\right)$. Furthermore, the equality $\lambda_{1}(\Omega, v)=F_{n}\left(|\Omega|,\|v\|_{\infty}\right)$ holds if and only if, up to translation, $\Omega=\Omega^{*}$ and $v=\|v\|_{\infty} e_{r}$. The function $F_{n}$ is defined by

$$
F_{n}(m, \tau):=\lambda_{1}\left(B_{R_{n, m}}^{n}, \tau e_{r}\right)
$$

where $B_{R_{n, m}}^{n}$ denotes the open Euclidean ball of $\mathbb{R}^{n}$ centered at 0 with radius $R_{n, m}=$ $\left(m / \alpha_{n}\right)^{1 / n}$ (that is, $\left|B_{R_{n, m}}^{n}\right|=m$, with $\left.\alpha_{n}=\left|B_{1}^{n}\right|\right)$. The inequality (2.6) can then be viewed as an extension of (1.2) to the case of the Laplacian with a bounded drift (however, no explicit expression of $F_{n}(m, \tau)$ is available when $\tau>0$, unlike when $\left.\tau=0\right)$. Since the principal eigenvalues are non-increasing with respect to the inclusion of domains [7], the previous results can be reformulated as the following minimization problem: for every $n \geq 1$, $m>0$ and $\tau \geq 0$,

$$
\min _{|\Omega| \leq m,\|v\|_{L^{\infty}\left(\Omega, \mathbb{R}^{n}\right) \leq \tau}} \lambda_{1}(\Omega, v)=\lambda_{1}\left(B_{R_{n, m}}^{n}, \tau e_{r}\right)
$$

and the couple $\left(B_{R_{n, m}}^{n}, \tau e_{r}\right)$ is the unique minimizer (up to translation), among all the $C^{2}$ bounded domains $\Omega \subset \mathbb{R}^{n}$ with Lebesgue measure less or equal to $m$ and the vector fields $v \in L^{\infty}\left(\Omega, \mathbb{R}^{n}\right)$ with $\|v\|_{L^{\infty}\left(\Omega, \mathbb{R}^{n}\right)} \leq \tau$.

Finally, notice that the variational formula (1.3) for the Laplacian cannot be extended to the non-symmetric operator $-\Delta+v \cdot \nabla$, even if min-max integral [18] or pointwise [7] variational formulas exist, but are less easily useable. The proof of Theorem 2.6 does not rely on the Schwarz rearrangement but on a different symmetrization technique, which will be described in Section 4. Before doing so, we first consider in the next section several minimization problems for more general elliptic operators of the type $-\operatorname{div}(A \nabla)+v \cdot \nabla+V$. 


\section{Second order elliptic operators}

In this section, we consider more general elliptic operators

$$
-\operatorname{div}(A \nabla)+v \cdot \nabla+V
$$

on $C^{2}$ bounded domains $\Omega \subset \mathbb{R}^{n}$ under Dirichlet boundary condition. We assume that $A \in W^{1, \infty}\left(\Omega, \mathcal{S}_{n}(\mathbb{R})\right)$, where $\mathcal{S}_{n}(\mathbb{R})$ denotes the space of symmetric real-valued matrices of size $n \times n$. We also assume that the operator is uniformly elliptic, in the sense that

$$
\inf _{x \in \Omega, \xi \in \mathbb{R}^{n},|\xi|=1} A(x) \xi \cdot \xi>0 .
$$

For any such matrix field $A$, there are some ellipticity functions $\Lambda \in L_{+}^{\infty}(\Omega):=\left\{f \in L^{\infty}(\Omega)\right.$, inf $\left.\operatorname{ess}_{\Omega} f>0\right\}$ such that

$$
A \geq \Lambda I_{n} \text { a.e. in } \Omega
$$

in the sense of symmetric matrices, where $I_{n}$ denotes the identity matrix of size $n \times n$. The above condition is fulfilled for instance when, for a.e. $x \in \Omega, \Lambda(x)$ is the smallest eigenvalue of the matrix $A(x)$. The vector field $v: \Omega \rightarrow \mathbb{R}^{n}$ and the scalar potential $V: \Omega \rightarrow \mathbb{R}$ are assumed to be bounded. Under these general assumptions, the operator $-\operatorname{div}(A \nabla)+v \cdot \nabla+V$ under Dirichlet boundary condition has a principal eigenvalue $\lambda_{1}(\Omega, A, v, V)$ associated with a corresponding eigenfunction $\varphi$ which belongs to the Sobolev spaces $W^{2, p}(\Omega)$ for all $1 \leq p<+\infty$ and satisfies

$$
\left\{\begin{aligned}
-\operatorname{div}(A \nabla \varphi)+v \cdot \nabla \varphi+V \varphi & =\lambda_{1}(\Omega, A, v, V) \varphi & & \text { a.e. in } \Omega, \\
\varphi & >0 & & \text { in } \Omega, \\
\varphi & =0 & & \text { on } \partial \Omega,
\end{aligned}\right.
$$

see [7]. Furthermore, $\lambda_{1}(\Omega, A, v, V)$ is simple, real and all other eigenvalues have a real part which is larger than $\lambda_{1}(\Omega, A, v, V)$. It is also known [7] that $\lambda_{1}(\Omega, A, v, V)>\inf \operatorname{ess}_{\Omega} V$ and that $\lambda_{1}(\Omega, A, v, V)>0$ if and only if the operator $-\operatorname{div}(A \nabla)+v \cdot \nabla+V$ satisfies the maximum principle.

The aim of the following results is to show that, to any such operator

$$
L=-\operatorname{div}(A \nabla)+v \cdot \nabla+V
$$

in $\Omega$ under Dirichlet boundary condition, we can associate an operator

$$
L^{*}=-\operatorname{div}\left(A^{*} \nabla\right)+v^{*} \cdot \nabla+V^{*}
$$

in $\Omega^{*}$ under Dirichlet boundary condition on $\partial \Omega^{*}$, in such a way that:

- the coefficients of $L^{*}$ are radially symmetric,

- some quantities depending on the coefficients (averages, uniform bounds, distribution functions, determinant and another symmetric function of the eigenvalues of the second order coefficients) are preserved, 
- the principal eigenvalue of $L^{*}$ is not larger than the principal eigenvalue of $L$, that is

$$
\lambda_{1}\left(\Omega^{*}, A^{*}, v^{*}, V^{*}\right) \leq \lambda_{1}(\Omega, A, v, V) .
$$

Let us start with the case of $L^{\infty}$ constraints on $v$ and $V$ and an $L^{1}$ constraint on $\Lambda^{-1}$.

Theorem 3.1 [15] Let $\Omega \subset \mathbb{R}^{n}$ be a $C^{2}$ bounded domain which is not a ball. Assume that $\|A\|_{W^{1, \infty}\left(\Omega, \mathcal{S}_{n}(\mathbb{R})\right)} \leq M$, that $A \geq \Lambda I_{n}$ a.e. in $\Omega$, that $\inf \operatorname{ess}_{\Omega} \Lambda \geq m>0$, that $\|v\|_{L^{\infty}\left(\Omega, \mathbb{R}^{n}\right)} \leq$ $\tau$ and that $\inf \operatorname{ess}_{\Omega} V \geq \kappa$. Then there exists a positive constant $\eta>0$, which depends only on $n, \Omega, M, m$ and $\tau$, and a positive radially symmetric function $\Lambda^{*} \in C^{\infty}\left(\overline{\Omega^{*}}\right)$ such that

$$
\inf _{\Omega} \operatorname{ess} \Lambda \leq \Lambda^{*} \leq \sup _{\Omega} \operatorname{ess} \Lambda \text { in } \overline{\Omega^{*}}, \quad\left\|\left(\Lambda^{*}\right)^{-1}\right\|_{L^{1}\left(\Omega^{*}\right)}=\left\|\Lambda^{-1}\right\|_{L^{1}(\Omega)}
$$

and

$$
\lambda_{1}\left(\Omega^{*}, \Lambda^{*} I_{n}, \tau e_{r}, \kappa\right) \leq \lambda_{1}(\Omega, A, v, V)-\eta .
$$

This result means that the principal eigenvalue of the operator $-\operatorname{div}\left(\Lambda^{*} \nabla\right)+\tau e_{r} \cdot \nabla+\kappa$ in $\Omega^{*}$ under Dirichlet boundary condition on $\partial \Omega^{*}$ is strictly smaller than that of the operator $-\operatorname{div}(A \nabla)+v \cdot \nabla+V$ in $\Omega$ under Dirichlet boundary condition on $\partial \Omega$. Furthermore, the gap is quantified by a positive real number $\eta$ which depends only on some bounds on the coefficients and on $\Omega$, through the isoperimetric defect $\mathcal{H}_{n-1}(\partial \Omega) / \mathcal{H}_{n-1}\left(\partial \Omega^{*}\right)-1>0$, where $\mathcal{H}_{n-1}$ denotes the $n$-1-dimensional Hausdorff measure. We have assumed that $A \geq \Lambda I_{n}$ a.e. in $\Omega$, where the function $\Lambda$ may not be constant in general. Actually, it turns out from the proof of the result that, by construction, the function $\Lambda^{*}$ is not constant in general, that is the principal part $-\operatorname{div}\left(\Lambda^{*} \nabla\right)$ is not proportional to the Laplacian in general. However, when $\Lambda$ is taken as a positive constant (this is of course always possible), then $\Lambda^{*}$ is then equal to the same constant in $\Omega^{*}$ and, in this case, the conclusion of Theorem 3.1 easily recovers that of Theorem 2.6 when $\Omega$ is not a ball. The fact that the $L^{1}$ norms of the inverses of $\Lambda$ and $\Lambda^{*}$ are equal also comes from the proof and the construction of $\Lambda^{*}$, which is defined as a pseudo-rearrangement of $\Lambda$ through the level sets of $\varphi$, see Section 4 .

When $\Omega$ is a ball, then the inequality (3.8) holds without the coefficient $\eta$, even if it means that $\Lambda^{*}$ is not smooth anymore in general. Finally, one obtains a Faber-Krahn type inequality, that is, for all $n \geq 1$, there is a function $G_{n}:(0,+\infty) \times(0,+\infty) \times[0,+\infty) \times \mathbb{R} \rightarrow \mathbb{R}$ such that

$$
\lambda_{1}(\Omega, A, v, V) \geq G_{n}\left(|\Omega|, \underset{\Omega}{\inf } \operatorname{ess} \Lambda[A],\|v\|_{L^{\infty}\left(\Omega, \mathbb{R}^{n}\right)}, \underset{\Omega}{\inf \operatorname{ess} V)}\right.
$$

for all $\Omega, A, v$ and $V$ as above, where, for a.e. $x \in \Omega, \Lambda[A(x)]$ denotes the smallest eigenvalue of the symmetric matrix $A(x)$, and

$$
G_{n}(m, \gamma, \tau, \kappa)=\lambda_{1}\left(B_{R_{n, m}}^{n}, \gamma I_{n}, \tau e_{r}, \kappa\right)=\lambda_{1}\left(B_{R_{n, m}}^{n}, \gamma I_{n}, \tau e_{r}, 0\right)+\kappa
$$

for all $m>0, \gamma>0, \tau \geq 0$ and $\kappa \in \mathbb{R}$. Furthermore, the inequality (3.9) is strict if $\Omega$ is not a ball. 
Remark 3.2 In general, the operators $L$ and $L^{*}$ given in Theorem 3.1 are not symmetric and, as already mentioned, no easily useable variational formulation for $\lambda_{1}(\Omega, A, v, V)$ exists, unlike (1.3) for the Laplacian. Therefore, the Schwarz symmetrization does not provide any immediate lower bound for $\lambda_{1}(\Omega, A, v, V)$ in terms of the principal eigenvalue of an elliptic operator in $\Omega^{*}$ whose coefficients stay in the same class of constraints. Even when $L$ is symmetric, the Schwarz rearrangement cannot be used to prove Theorem 3.1.

There are other results of the same kind with other types of constraints on the coefficients. Namely, the following results deal either with some integral constraints on $\Lambda$ and $v$ and some constraints on the distribution function of the negative part $V^{-}=\max (-V, 0)$ of $V$, or with some geometric constraints on the determinant and the trace of the matrices $A(x)$. For a function $f \in L^{\infty}(\Omega)$, its distribution function $\mu_{f}: \mathbb{R} \rightarrow[0,+\infty)$ is defined by

$$
\mu_{f}(t)=|\{x \in \Omega, f(x)>t\}| .
$$

Theorem 3.3 [15] Let $\Omega \subset \mathbb{R}^{n}$ be a $C^{2}$ bounded domain, $A \in W^{1, \infty}\left(\Omega, \mathcal{S}_{n}(\mathbb{R})\right), \Lambda \in L_{+}^{\infty}(\Omega)$, $v \in L^{\infty}\left(\Omega, \mathbb{R}^{n}\right)$ and $V: \bar{\Omega} \rightarrow \mathbb{R}$ be continuous. Assume that $A \geq \Lambda I_{n}$ a.e. in $\Omega$, and that $\lambda_{1}(\Omega, A, v, V) \geq 0$. Then, for every $\varepsilon>0$, there exist two radially symmetric $C^{\infty}\left(\overline{\Omega^{*}}\right)$ fields $\Lambda^{*}>0$ and $\omega^{*} \geq 0$, and a radially symmetric function $V^{*} \leq 0$ in $L^{\infty}\left(\Omega^{*}\right)$, such that

$$
\left\{\begin{array}{l}
\inf _{\Omega} \operatorname{ess} \Lambda \leq \Lambda^{*} \leq \sup _{\Omega} \operatorname{ess} \Lambda \text { in } \overline{\Omega^{*}}, \quad\left\|\left(\Lambda^{*}\right)^{-1}\right\|_{L^{1}\left(\Omega^{*}\right)}=\left\|\Lambda^{-1}\right\|_{L^{1}(\Omega)}, \\
\left\|\omega^{*}\right\|_{L^{\infty}\left(\Omega^{*}\right)} \leq\|v\|_{L^{\infty}\left(\Omega, \mathbb{R}^{n}\right)},\left\|\left(\omega^{*}\right)^{2}\left(\Lambda^{*}\right)^{-1}\right\|_{L^{1}\left(\Omega^{*}\right)}=\left\||v|^{2} \Lambda^{-1}\right\|_{L^{1}(\Omega)}, \\
\mu_{V^{*}}=\mu_{-V^{-}},
\end{array}\right.
$$

and

$$
\lambda_{1}\left(\Omega^{*}, \Lambda^{*} I_{n}, \omega^{*} e_{r}, V^{*}\right) \leq \lambda_{1}(\Omega, A, v, V)+\varepsilon .
$$

In the above inequality, the fields $\Lambda^{*}$ and $\omega^{*}$ are of class $C^{\infty}\left(\overline{\Omega^{*}}\right)$. But the price to pay is the presence of a positive $\varepsilon$ term (the fields depend on $\varepsilon$ ). Without the $\varepsilon$ term, an inequality can still be obtained, but the coefficients of the symmetrized operator in the ball $\Omega^{*}$ are not smooth anymore in general. More precisely, under the assumptions of Theorem 3.3, there are some radially symmetric bounded functions $\Lambda_{0}^{*} \in L_{+}^{\infty}\left(\Omega^{*}\right), \omega_{0}^{*} \geq 0$ and $V_{0}^{*} \leq 0$ such that

$$
\left\{\begin{array}{l}
\inf _{\Omega} \operatorname{ess} \Lambda \leq \Lambda_{0}^{*} \leq \sup _{\Omega} \operatorname{ess} \Lambda \text { a.e. in } \Omega^{*}, \quad\left\|\left(\Lambda_{0}^{*}\right)^{-1}\right\|_{L^{1}\left(\Omega^{*}\right)}=\left\|\Lambda^{-1}\right\|_{L^{1}(\Omega)}, \\
\left\|\omega_{0}^{*}\right\|_{L^{\infty}\left(\Omega^{*}\right)} \leq\|v\|_{L^{\infty}\left(\Omega, \mathbb{R}^{n}\right)}, \\
\left\|V_{0}^{*}\right\|_{L^{p}\left(\Omega^{*}\right)} \leq\left\|V^{-}\right\|_{L^{p}(\Omega)} \text { for all } 1 \leq p \leq+\infty,\left\|V_{0}^{*}\right\|_{L^{1}\left(\Omega^{*}\right)}=\left\|V^{-}\right\|_{L^{1}(\Omega)},
\end{array}\right.
$$

and

$$
\lambda_{1}\left(\Omega^{*}, \Lambda_{0}^{*} I_{n}, \omega_{0}^{*} e_{r}, V_{0}^{*}\right) \leq \lambda_{1}(\Omega, A, v, V) .
$$

Here, $\lambda_{1}\left(\Omega^{*}, \Lambda_{0}^{*} I_{n}, \omega_{0}^{*} e_{r}, V_{0}^{*}\right)$ is understood in a weak sense.

In the case when $\Omega \subset \mathbb{R}^{n}$ is not a ball, then a more precise quantified inequality can be established. Namely, for any constants $M>0, m>0, \tau \geq 0$ and $\kappa \geq 0$, there is a positive constant $\theta$ depending only on $\Omega, n, M, m, \tau$ and $\kappa$ such that the following holds: if 
$\|A\|_{W^{1, \infty}\left(\Omega, \mathcal{S}_{n}(\mathbb{R})\right)} \leq M, A \geq \Lambda I_{n}$ a.e. in $\Omega$, inf $\operatorname{ess}_{\Omega} \Lambda \geq m,\|v\|_{L^{\infty}\left(\Omega, \mathbb{R}^{n}\right)} \leq \tau,\|V\|_{L^{\infty}(\Omega)} \leq \kappa$ and $\lambda_{1}(\Omega, A, v, V)>0$, then there are two radially symmetric $C^{\infty}\left(\overline{\Omega^{*}}\right)$ fields $\Lambda^{*}>0$ and $\omega^{*} \geq 0$, and a radially symmetric function $V^{*} \leq 0$ in $L^{\infty}\left(\Omega^{*}\right)$ satisfying (3.10) and

$$
\lambda_{1}\left(\Omega^{*}, \Lambda^{*} I_{n}, \omega^{*} e_{r}, V^{*}\right) \leq \frac{\lambda_{1}(\Omega, A, v, V)}{1+\theta} .
$$

The last theorem is concerned with a class of constraints on the trace $\operatorname{Tr}(A)$ and the determinant $\operatorname{Det}(A)$ of the matrix field $A$.

Theorem 3.4 [15] Under the general conditions of Theorem 3.3, assume moreover that $n \geq 2$ and let $\rho>0$ and $\sigma>0$ be such that

$$
\operatorname{Det}(A) \geq \rho \text { and } \operatorname{Tr}(A) \leq \sigma \text { a.e. in } \Omega .
$$

Then there are some constants $0<a_{1} \leq a_{2}$ and a matrix field $A^{*} \in C^{\infty}\left(\overline{\Omega^{*}} \backslash\{0\}, \mathcal{S}_{n}(\mathbb{R})\right)$ depending only on $n, \rho, \sigma$, such that $A \geq a_{1} I_{n}$ a.e. in $\Omega$,

$$
\left\{\begin{array}{l}
A^{*} \geq a_{1} I_{n} \text { in } \overline{\Omega^{*}} \backslash\{0\}, \operatorname{Det}\left(A^{*}\right)=\rho, \operatorname{Tr}\left(A^{*}\right)=\sigma \text { in } \overline{\Omega^{*}} \backslash\{0\}, \\
A^{*}(x) x \cdot x=a_{1}|x|^{2} \text { and } A^{*}(x) y \cdot y=a_{2}|y|^{2} \text { for all } y \perp x \text { and } x \in \overline{\Omega^{*}} \backslash\{0\},
\end{array}\right.
$$

and, for every $\varepsilon>0$, there exist a radially symmetric function $\omega^{*} \geq 0$ in $C^{\infty}\left(\overline{\Omega^{*}}\right)$ and a radially symmetric function $V^{*} \leq 0$ in $L^{\infty}\left(\Omega^{*}\right)$ satisfying

$$
\left\{\begin{array}{l}
\left\|\omega^{*}\right\|_{L^{\infty}\left(\Omega^{*}\right)} \leq\|v\|_{L^{\infty}\left(\Omega, \mathbb{R}^{n}\right)}, \quad\left\|\omega^{*}\right\|_{L^{2}\left(\Omega^{*}\right)}=\|v\|_{L^{2}\left(\Omega, \mathbb{R}^{n}\right)}, \\
\mu_{V^{*}}=\mu_{-V^{-}},
\end{array}\right.
$$

and

$$
\lambda_{1}\left(\Omega^{*}, A^{*}, \omega^{*} e_{r}, V^{*}\right) \leq \lambda_{1}(\Omega, A, v, V)+\varepsilon .
$$

Remark 3.5 The same result holds when the trace $\operatorname{Tr}(A)$ is replaced by any symmetric function of order $1 \leq p \leq n-1$ of the eigenvalues of the matrix $A$.

Let us now comment the previous results. First, when the function $\Lambda$ is taken as a positive constant $\gamma$ and when $V \geq 0$ (whence $\lambda_{1}(\Omega, A, v, V)>0$ ), then the proof of Theorem 3.3 implies that

$$
\lambda_{1}(\Omega, A, v, V) \geq \lambda_{1}\left(\Omega^{*}, \gamma I_{n},\|v\|_{L^{\infty}\left(\Omega, \mathbb{R}^{n}\right)} e_{r}, 0\right) .
$$

This last inequality could also be derived implicitly from results of Talenti [26] and extraarguments based on the strong maximum principle as in [7]. Talenti's results are concerned with comparisons of the solutions of some elliptic equations in $\Omega$ with the solutions of some elliptic equations in $\Omega^{*}$ with symmetrized coefficients (see also [3, 27]). Talenti's proof is based on the Schwarz symmetrization and uses the fact that the principal part $\operatorname{div}(A \nabla)$ of the operator in $\Omega$ is compared with a constant times the Laplacian in $\Omega^{*}$. In particular, if one takes $A=I_{n}, \Lambda=1$ and $V=0$, then the aforementioned references provide the inequality (2.5), the case of equality being actually more intricate and new. In particular, 
the proofs of the quantified improved inequalities (3.8) and (3.12) when $\Omega$ is not a ball require special technical extra-arguments and are completely new, even in the simple case when $\Lambda$ is a constant. But we again point out that the function $\Lambda$ in Theorems 3.1 and 3.3 may not be chosen to be a constant and the function $\Lambda^{*}$ is not constant either in general. In other words, the principal part $\operatorname{div}\left(\Lambda^{*} \nabla\right)$ of the symmetrized operator in $\Omega^{*}$ is not proportional to the Laplacian in general.

The general conclusion of all above theorems is that when we want to minimize the principal eigenvalues of elliptic operators in a domain with a fixed Lebesgue measure, under the aforementioned constraints, we can restrict ourselves to the case of operators with radially symmetric coefficients in an equimeasurable ball. We point out that, in their generality, the above results are actually new in dimension 1 and in the case of symmetric operators (with $v=0$ ). Moreover, the case of operators with constraints on the determinant and another symmetric function of the eigenvalues of the diffusion matrices $A$ had not been considered before. The proofs require brand new techniques, which will be described in the next section.

\section{A symmetrization technique}

The proofs of the results presented in Sections 2 and 3 are based on a new symmetrization technique, which is different from the Schwarz symmetrization, and which has its own interest, independently of the eigenvalue problems. Let us now describe the main lines of this technique and the key-results associated with it.

Let $\Omega$ be a $C^{2}$ bounded domain of $\mathbb{R}^{n}$ and let $\varphi$ be a $C^{2}(\Omega) \cap W^{2, p}(\Omega)$ (for all $1 \leq p<+\infty$ ) function such that

$$
\varphi>0 \text { in } \Omega \text { and } \nabla \varphi \neq 0, \varphi=0 \text { on } \partial \Omega .
$$

The function $\varphi$ then belongs to the Hölder spaces $C^{1, \alpha}(\bar{\Omega})$ for all $0 \leq \alpha<1$. Call $M=$ $\max _{\bar{\Omega}} \varphi>0$. Assume now that the set

$$
Z=\{a>0, \exists x \in \Omega, \varphi(x)=a, \nabla \varphi(x)=0\}
$$

of critical values of $\varphi$ in $\Omega$ is finite, and let $Y=[0, M] \backslash Z$ be the set of non-critical values of $\varphi$. For all $a \in[0, M)$, define $\Omega_{a}$ as the upper level set of $\varphi$ with level $a$, that is

$$
\Omega_{a}=\{x \in \Omega, \varphi(x)>a\}
$$

and, for all $a \in[0, M]$,

$$
\Sigma_{a}=\{x \in \bar{\Omega}, \varphi(x)=a\} .
$$

Assume that, for all $0 \leq a \leq M,\left|\Sigma_{a}\right|=0$. Let $R=R_{n,|\Omega|}$ be the radius of $\Omega^{*}$. For all $a \in[0, M)$, define $\rho(a) \in(0, R]$ such that

$$
\left|\Omega_{a}\right|=\left|B_{\rho(a)}^{n}\right|=\alpha_{n} \rho(a)^{n},
$$

and set $\rho(M)=0$. It is easy to check that the function $\rho:[0, M] \rightarrow[0, R]$ is continuous, decreasing, one-to-one and onto. Lastly, define

$$
E=\left\{x \in \overline{\Omega^{*}},|x| \in \rho(Y)\right\} .
$$


Given $\varphi$ as above, let now $A$ be a $C^{1}\left(\bar{\Omega}, \mathcal{S}_{n}(\mathbb{R})\right)$ matrix field such that $A \geq \Lambda I_{n}$ in $\bar{\Omega}$, where $\Lambda$ is a $C^{1}(\bar{\Omega})$ function with $\min _{\bar{\Omega}} \Lambda>0$. For all $x \in E$, set

$$
\widehat{\Lambda}(x)=\frac{\int_{\Sigma_{\rho^{-1}(|x|)}}|\nabla \varphi(y)|^{-1} d \sigma_{\rho^{-1}(|x|)}(y)}{\int_{\Sigma_{\rho^{-1}(|x|)}} \Lambda(y)^{-1}|\nabla \varphi(y)|^{-1} d \sigma_{\rho^{-1}(|x|)}(y)}=: F(|x|)>0,
$$

where $d \sigma_{\rho^{-1}(|x|)}$ denotes the surface measure on $\Sigma_{\rho^{-1}(|x|)}$. One has $\min _{\bar{\Omega}} \Lambda \leq \widehat{\Lambda} \leq \max _{\bar{\Omega}} \Lambda$ a.e. in $\Omega^{*}$. It follows from the co-area formula that $\left\|\widehat{\Lambda}^{-1}\right\|_{L^{1}\left(\Omega^{*}\right)}=\|\Lambda\|_{L^{1}(\Omega)}$. The function $\widehat{\Lambda}$ is a pseudo-rearrangement of the function $\Lambda$ with respect to the level sets of $\varphi$, as in $[1,2]$.

Given $\varphi, A, \Lambda$ and $\widehat{\Lambda}$ as above, we now define the radially symmetric function $\widehat{\varphi} \in$ $C\left(\overline{\Omega^{*}}\right) \cap H_{0}^{1}\left(\Omega^{*}\right) \cap W^{1, \infty}\left(\Omega^{*}\right)$ as the unique radially decreasing function, positive in $\Omega^{*}$ such that the integrals of the second order operators $\operatorname{div}(A \nabla)$ and $\operatorname{div}(\widehat{\Lambda} \nabla)$ applied to $\varphi$ and $\widehat{\varphi}$ on the corresponding equimeasurable sets of $\Omega$ and $\Omega^{*}$ are equal, that is

$$
\int_{\Omega_{a}} \operatorname{div}(A \nabla \varphi)(x) d x=\int_{B_{\rho(a)}} \operatorname{div}(\widehat{\Lambda} \nabla \widehat{\varphi})(x) d x
$$

for all $0 \leq a<M$. In other words we set, for all $x \in \overline{\Omega^{*}}$,

$$
\widehat{\varphi}(x)=-\int_{|x|}^{R} G(r) d r
$$

where

$$
\begin{aligned}
G(r) & =\frac{1}{n \alpha_{n} r^{n-1} F(r)} \int_{\Omega_{\rho^{-1}(r)}} \operatorname{div}(A \nabla \varphi)(z) d z \\
& =\frac{-1}{n \alpha_{n} r^{n-1} F(r)} \int_{\Sigma_{\rho^{-1}(r)}}\left(A(y) \nu_{\rho^{-1}(r)}(y) \cdot \nu_{\rho^{-1}(r)}(y)\right)|\nabla \varphi(y)| d \sigma_{\rho^{-1}(r)}(y)<0
\end{aligned}
$$

for all $r \in \rho(Y)$, where $\nu_{\rho^{-1}(r)}$ denotes the outward unit normal to $\Omega_{\rho^{-1}(r)}$ on $\Sigma_{\rho^{-1}(r)}$. The function $\widehat{\varphi}$ is then actually of class $C^{1}$ in $E \cup\{0\}$ and $C^{2}$ in $E \cap \Omega^{*}$.

The first key-point of this symmetrization is the comparison between $\varphi$ and $\widehat{\varphi}$ on the sets $\Sigma_{a}$ and $\partial B_{\rho(a)}^{n}$ for all $a \in[0, M]$.

Theorem $4.1[15]$ For all $x \in \overline{\Omega^{*}}$ and $y \in \bar{\Omega}$ such that $|x|=\rho(\varphi(y))$ (that is $\rho^{-1}(|x|)=$ $\varphi(y))$, there holds

$$
\widehat{\varphi}(x) \geq \varphi(y)
$$

The second key-point is a pointwise partial differential inequality involving $\operatorname{div}(A \nabla \varphi)$ and $\operatorname{div}(\widehat{\Lambda} \nabla \widehat{\varphi})$, as well as some first and zeroth order terms which we define below. Given $\varphi, A, \Lambda, \widehat{\Lambda}$ and $\widehat{\varphi}$ as above, let now $v: \bar{\Omega} \rightarrow \mathbb{R}^{n}$ and $V: \bar{\Omega} \rightarrow \mathbb{R}$ be two given continuous 
fields. Let us then define a symmetrized drift $\widehat{v}$ and a symmetrized potential $\widehat{V}$ in $\Omega^{*}$. For all $x \in E$, set

$$
\widehat{v}(x)=\left(\frac{\int_{\Sigma_{\rho^{-1}(|x|)}}|v(y)|^{2} \Lambda(y)^{-1}|\nabla \varphi(y)|^{-1} d \sigma_{\rho^{-1}(|x|)}(y)}{\int_{\Sigma_{\rho^{-1}(|x|)}} \Lambda(y)^{-1}|\nabla \varphi(y)|^{-1} d \sigma_{\rho^{-1}(|x|)}(y)}\right)^{1 / 2} e_{r}(x) .
$$

One has $\|\widehat{v}\|_{L^{\infty}\left(\Omega^{*}, \mathbb{R}^{n}\right)} \leq\|v\|_{L^{\infty}\left(\Omega, \mathbb{R}^{n}\right)}$, and $\left\||\widehat{v}|^{2} \widehat{\Lambda}^{-1}\right\|_{L^{1}\left(\Omega^{*}\right)}=\left\||v|^{2} \Lambda^{-1}\right\|_{L^{1}(\Omega)}$ from the co-area formula. Finally, for all $x \in E$, define

$$
\widehat{V}(x)=\frac{-\int_{\Sigma_{\rho^{-1}(|x|)}} V^{-}(y)|\nabla \varphi(y)|^{-1} d \sigma_{\rho^{-1}(|x|)}(y)}{\int_{\Sigma_{\rho^{-1}(|x|)}}|\nabla \varphi(y)|^{-1} d \sigma_{\rho^{-1}(|x|)}(y)} \leq 0 .
$$

Theorem 4.2 [15] For all $x \in E \cap \Omega^{*}$, there exists $y \in \Omega$ such that $\varphi(y)=\rho^{-1}(|x|)$, that is $y \in \Sigma_{\rho^{-1}(|x|)}$, and

$$
\begin{aligned}
-\operatorname{div}(\widehat{\Lambda} \nabla \widehat{\varphi})(x)+\widehat{v}(x) & \cdot \nabla \widehat{\varphi}(x)+\widehat{V}(x) \widehat{\varphi}(x) \\
& \leq-\operatorname{div}(A \nabla \varphi)(y)+v(y) \cdot \nabla \varphi(y)+V(y) \varphi(y) .
\end{aligned}
$$

Theorems 4.1 and 4.2 are fundamental in the proofs of the main results stated in the previous sections. However, they have their own interest which is independent from the eigenvalue problems, since they are only related to the symmetrization technique of the function $\varphi$ and of the coefficients $\Lambda, v$ and $V$. The proofs of the two inequalities stated in Theorems 4.1 and 4.2 use, in particular, the co-area formula, the Cauchy-Schwarz inequality and the classical isoperimetric inequality in $\mathbb{R}^{n}$ (recall that $\left|\Omega_{a}\right|=\left|B_{\rho(a)}^{n}\right|$ for all $a \in[0, M]$, whence $\left.\mathcal{H}_{n-1}\left(\Sigma_{a}\right) \geq \mathcal{H}_{n-1}\left(B_{\rho(a)}^{n}\right)=n \alpha_{n} \rho(a)^{n-1}\right)$.

Finally, given these two theorems, let us sketch the proof of one of the main results stated in the previous sections, say the inequality (3.11), at least in a rough sense without the $\varepsilon$ term. Given $\varphi, A, \Lambda, \widehat{\Lambda}, \widehat{\varphi}, v, V, \widehat{v}$ and $\widehat{V}$ as above, let us now assume that $\varphi$ solves (3.7), that is $\varphi$ is the principal eigenvalue associated with the operator $-\operatorname{div}(A \nabla)+v \cdot \nabla+V$ in $\Omega$ under Dirichlet boundary condition on $\partial \Omega$. Assume that the principal eigenvalue $\lambda:=\lambda_{1}(\Omega, A, v, V)$ is nonnegative. It follows from Theorems 4.1 and 4.2 that, for all $x \in E \cap \Omega^{*}$,

$$
-\operatorname{div}(\widehat{\Lambda} \nabla \widehat{\varphi})(x)+\widehat{v}(x) \cdot \nabla \widehat{\varphi}(x)+\widehat{V}(x) \widehat{\varphi}(x) \leq \lambda \varphi(y) \leq \lambda \widehat{\varphi}(x)
$$

for some $y \in \Sigma_{\rho^{-1}(|x|)}$. If $\widehat{\Lambda}$ and $\widehat{\varphi}$ were of class $C^{1}(\bar{\Omega})$ and $C^{2}(\Omega)$ respectively, it would then follow from the strong maximum principle and from Hopf lemma as in [7] that

$$
\lambda_{1}\left(\Omega^{*}, \widehat{\Lambda} I_{n}, \widehat{v}, \widehat{V}\right) \leq \lambda
$$


which is almost the required conclusion (3.11) in Theorem 3.3.

However, in general, the set of critical values of $\varphi$ in $\Omega$ is not finite (it would be the case if $\varphi$ were real analytic in $\Omega$ ) and the coefficients of the operator $-\operatorname{div}(A \nabla)+v \cdot \nabla+V$ and the function $\varphi$ itself are not smooth enough. The complete proof of the inequality (3.11) is done by smooth approximations of the coefficients of the operator (by preserving the constraints) and of the function $\varphi$. It involves many technicalities.

When $\Omega$ is not a ball, improved versions of the previous auxiliary and final inequalities can be established. In particular, the precised versions of the inequalities stated in Theorems 4.1 and 4.2 are proved to be independent of the approximation process, and they rely on the fact that $\mathcal{H}_{n-1}(\partial \Omega)>\mathcal{H}_{n-1}\left(\partial \Omega^{*}\right)$ and $\nabla \varphi \neq 0$ on $\partial \Omega$. We refer to [15] for complete proofs of the theorems stated in Sections 2, 3 and 4 and further results and comments.

\section{References}

[1] A. Alvino and G. Trombetti, A lower bound for the first eigenvalue of an elliptic operator, J. Math. Anal. Appl. 94 (1983), 328-337.

[2] A. Alvino and G. Trombetti, Isoperimetric inequalities connected with torsion problem and capacity, Boll. Union Mat. Ital. B 4 (1985), 773-787.

[3] A. Alvino, G. Trombetti, P.-L. Lions and S. Matarasso, Comparison results for solutions of elliptic problems via symmetrization, Ann. Inst. Henri Poincaré 162 (1999), 167-188.

[4] M.S. Ashbaugh and R.D. Benguria, A sharp bound for the ratio of the first two eigenvalues of Dirichlet Laplacians and extensions, Ann. Math. 135 (1992), 601-628.

[5] M.S. Ashbaugh and R.D. Benguria, On Rayleigh's conjecture for the clamped plate and its generalization to three dimensions, Duke Math. J. 78 (1995), 1-17.

[6] C. Bandle, Isoperimetric Inequalities and Applications, Pitman Monographs and Studies in Math. 7, Boston, 1980.

[7] H. Berestycki, L. Nirenberg and S.R.S. Varadhan, The principal eigenvalue and maximum principle for second-order elliptic operators in general domains, Comm. Pure Appl. Math. 47 (1994), 47-92.

[8] F. Brock, An isoperimetric inequality for eigenvalues of the Stekloff problem, $Z$. Angew. Math. Mech. 81 (2001), 69-71.

[9] D. Bucur and A. Henrot, Minimization of the third eigenvalue of the Dirichlet Laplacian, Proc. Royal Soc. London Ser. A 456 (2000), 985-996.

[10] S.-Y. Cheng and K. Oden, Isoperimetric inequalities and the gap between the first and second eigenvalues of an Euclidean domain, J. Geom. Anal. 7 (1997), 217-239. 
[11] D. Daners, A Faber-Krahn inequality for Robin problems in any space dimension, Math. Ann. 335 (2006), 767-785.

[12] D. Daners and J. Kennedy, Uniqueness in the Faber-Krahn inequality for Robin problems, SIAM J. Math. Anal. 39 (2007/08), 1191-1207.

[13] G. Faber, Beweis, dass unter allen homogenen Membranen von gleicher Fläche und gleicher Spannung die kreisförmige den tiefsten Grundton gibt, Sitzungsberichte der mathematisch-physikalischen Klasse der Bayerischen Akademie der Wissenschaften zu München (1923), 169-172.

[14] F. Hamel, N. Nadirashvili, E. Russ, An isoperimetric inequality for the principal eigenvalue of the Laplacian with drift, C. R. Acad. Sci. Paris Ser. I 340 (2005), 347-352.

[15] F. Hamel, N. Nadirashvili, E. Russ, Rearrangement inequalities and applications to isoperimetric problems for eigenvalues, Ann. Math. 174 (2011), 647-755.

[16] A. Henrot, Minimization problems for eigenvalues of the Laplacian, J. Evol. Eq. 3 (2003), 443-461.

[17] A. Henrot, Extremum Problems for Eigenvalues of Elliptic Operators, Birkhäuser, 2006.

[18] C.J. Holland, A minimum principle for the principal eigenvalue for second-order linear elliptic equations with natural boundary conditions, Comm. Pure Appl. Math. 31 (1978), 509-519.

[19] E. Krahn, Über eine von Rayleigh formulierte Minimaleigenschaft des Kreises, Math. Ann. 94 (1925), 97-100.

[20] E. Krahn, Über Minimaleigenschaft der Kugel in drei und mehr Dimensionen, Acta Comm. Univ. Tartu (Dorpat) A9 (1926), 1-44.

[21] N.S. Nadirashvili, Rayleigh's conjecture on the principal frequency of the clamped plate, Arch. Ration. Mech. Anal. 129 (1995), 1-10.

[22] L.E. Payne, G. Pólya and H.F. Weinberger, On the ratio of consecutive eigenvalues, J. Math. Phys. 35 (1956), 289-298.

[23] G. Pólya and G. Szegö, Isoperimetric Inequalities in Mathematical Physics, Annals of Mathematics Studies 27, Princeton Univ. Press, Princeton, 1951.

[24] J.W.S. Rayleigh, The Theory of Sound, $2^{\text {nd }}$ ed. revised and enlarged (in 2 vols.), Dover Publications, New York, 1945 (republication of the 1894/1896 edition).

[25] G. Szegö, Inequalities for certain eigenvalues of a membrane of given area, J. Rational Mech. Anal. 3 (1954), 343-356. 
[26] G. Talenti, Linear elliptic P.D.E.'s: level sets, rearrangements and a priori estimates of solutions, Boll. Union Mat. Ital. B 6 (1985), 917-949.

[27] G. Trombetti and J. L. Vazquez, A symmetrization result for elliptic equations with lower-order terms, Ann. Fac. Sci. Toulouse 7 (1985), 137-150.

[28] H.F. Weinberger, An isoperimetric inequality for the $N$-dimensional free membrane problem, J. Rational Mech. Anal. 5 (1956), 633-636. 